\title{
Características acústicas y psicosociales de mujeres con voces hiperagudas
}

\author{
Loreto Nercelles ${ }^{1}$, Joselyn Vidal ${ }^{2}$, Valeria Molina ${ }^{2}$, Karla Ruz $^{2}$, Carol Taiba ${ }^{2}$, Francisco Tocornal ${ }^{3}$
}

Recibido: 10 de julio de 2019 / Revisado: 20 de septiembre de 2019 / Aceptado :30 de octubre de 2019

Resumen: La voz hiperaguda en mujeres es un trastorno de la voz poco estudiado. El objetivo de esta investigación es establecer un perfil vocal de mujeres con voces hiperagudas y determinar las molestias psicosociales que puede desencadenar este síntoma comparándolo con mujeres con voz con frecuencia fundamental promedio. Mediante un diseño de casos y controles se estudió una muestra de 12 mujeres con frecuencia fundamental sobre los $250 \mathrm{~Hz}$. y se comparó con 24 mujeres de rangos de frecuencia fundamental dentro de la norma $(210-250 \mathrm{~Hz}$.). Además, para valorar las molestias psicosociales se les aplicó una encuesta de evaluación de la muda vocal (PEMUV). Se encontraron diferencias significativas entre ambos grupos en el parámetro frecuencia fundamental en vocal $/ \mathrm{a} /(\mathrm{p}=0.002)$, frecuencia fundamental en lenguaje automático $(\mathrm{p}=0.003)$, frecuencia fundamental en lenguaje espontáneo $(\mathrm{p}=0.002)$, jitter $(\mathrm{p}=0.05)$ y shimmer $(0.00)$. Además, en el análisis de la encuesta, si existieron diferencias significativas en 12 de las 14 preguntas de este instrumento. Por último, se codificaron las respuestas de la pregunta abierta, evidenciándose alteraciones familiares, laborales, frustración y confusión de edad debido a la voz hiperaguda. La presente investigación logró establecer un perfil de las mujeres con voces hiperagudas. De acuerdo con los resultados obtenidos, las mujeres con voz hiperaguda, presentan valoración negativa de la voz, molestias a nivel emocional y social.

Palabras clave: disfonía; puberfonía; voz; voz hiperaguda.

\section{[en] Acustic and psychosocial characteristics of women with high pitch voice}

Abstract: The high pitch voice in women is being poorly studied. The objective of this research is to establish a vocal profile of women with high pitch voices and determine the psychosocial discomfort that this symptom can trigger by comparing it with women with a voice with average fundamental frequency. Through a case-control design, a sample of 12 women with a fundamental frequency over $250 \mathrm{~Hz}$ was studied and compared with 24 women of fundamental frequency ranges within the norm (210-250 Hz.). In addition, in order to assess psychosocial discomforts, an evaluation survey (PEMUV) was applied. Significant differences were found between both groups in the fundamental frequency parameter in vowel / a / $(\mathrm{p}=0.002)$, fundamental frequency in automatic language $(\mathrm{p}=0.003)$, fundamental frequency in spontaneous language $(\mathrm{p}$ $=0.002)$, jitter $(\mathrm{p}=0.05)$ and shimmer $(0.00)$. In addition, in the analysis of the survey, there were significant differences in 12 out of 14 questions of this instrument. Finally, the answers to the open question were coded, evidencing family and work alterations, frustration and age confusion due to the high pitch. The present investigation managed to establish a profile of women with high pitch. According to the results obtained, women with high fundamental frequency present negative evaluation of the voice, as well as, emotional and social discomfort.

Keywords: dysphonia; high pitch voice; puberphonia; voice.

Sumario: Introducción. Metodología. Medición de variables. Resultados. Discusión. Bibliografía.

Cómo citar: Nercelles, L; et al. (2020) Características acústicas y psicosociales de mujeres con voces hiperagudas, Revista de investigación en Logopedia 10(1), 1-6.

\section{Introducción}

La voz hiperaguda en mujeres es un trastorno de la voz poco estudiado. Si bien existen investigaciones que permiten ubicar la voz dentro de rangos de normalidad según género y edad, no existe evidencia de las implicancias vocales y psicosociales de los desvíos hacia los agudos de frecuencia fundamental (F0) en mujeres (Aponte, 2003)

\footnotetext{
Escuela Fonoaudiología Universidad Andres Bello, Santiago de Chile.

loreto.nercelles@unab.cl

Escuela Fonoaudiología Universidad Santo Tomás, Santiago de Chile.

Servicio Otorrinolaringología, Clínica Las Condes, Santiago de Chile. 
La frecuencia fundamental en niños y niñas es bastante similar hasta alrededor de los 8 años, luego se inicia la muda vocal, donde el niño y la niña modifican sus valores de F0. Posteriormente en la etapa senil, la F0 vuelve a modificarse aumentando en hombres y disminuyendo en mujeres (Colton, Casper, \& Leonard, 2011).

Las mujeres modifican su voz alrededor de los 12 a 14 años (Behlau, 1995; Spiegel, Sataloff, \& Emerich, 1997). Durante el proceso de la muda vocal sólo descienden 3-4 semitonos en su frecuencia fundamental, en comparación a los varones quienes descienden una octava. Por esta razón, el cambio de voz en las mujeres es apenas percibido (Aponte, 2003; Banerjee, Eajlen, Meohurst, \& Murty, 1995; Brosé, 2018; Dinville, 1996).

Hay ocasiones en que el cambio de voz se retrasa o no se completa. A estas alteraciones se les llaman trastornos de la muda vocal o puberfonías. La sintomatología está dada en todos los casos por frecuencia fundamental más aguda de la que le corresponde por edad (Anelli, Costa, Duprat, \& Eckley, 1999; Fuchs, Fröehlich, Hentschel, Stuermer, Kruse, \& Knauft, 2007; Wojciechowska, Obrebowski, Studzinska, \& Swidzinski, 2010)

El impacto vocal y psicosocial de poseer un tono desviado a los agudos en la etapa adulta, ha sido más estudiado en hombres con puberfonía que en mujeres. El instrumento más utilizado para medir handicap vocal en voz hiperaguda es el Vocal Handicap Index (Denizoglu, Sahin, Bayrak, \& Uygun, 2018; Liang, Huang, Chen, Huang, Zhang, $\mathrm{Su}$, et al, 2017; Remacle, Matar, Verduyckt, \& Lawson, 2010). La pauta de Evaluación de la Muda Vocal (PEMUV) es otra herramienta que ha sido aplicada a varones con puberfonía. En una investigación realizada a 26 hombres con voces hiperagudas, se aplicó este instrumento a los que refirieron una valoración negativa de la voz y un sinnúmero de molestias a nivel emocional y social (Nercelles, 2018).

Dentro de las limitaciones encontradas en las revisiones y estudios de voz hiperaguda, se destaca la falta de información acerca de este trastorno en mujeres (Nercelles, 2019). Si bien la mayoría de las alteraciones según la bibliografía se dan en varones, sería interesante indagar qué ocurre en la evaluación y terapia de la voz femenina hiperaguda.

El objetivo de esta investigación es establecer un perfil vocal de mujeres con voces hiperagudas y determinar las molestias psicosociales que puede desencadenar este síntoma comparándola con mujeres de voces con frecuencia fundamental menor.

\section{Metodología}

Participantes: La muestra total estuvo compuesta por 12 mujeres con voces hiperagudas (F0 mayor a $250 \mathrm{~Hz}$.) y 24 mujeres con F0 dentro de la norma $(210-240 \mathrm{~Hz})$. El rango etario se encontró entre 18 y 45 años, promedio de 30 años de edad en los casos y 28 años en los controles.

Las mujeres a evaluar se seleccionaron mediante un muestreo no probabilístico. Para la obtención del grupo casos se planificó un llamado a través de prensa escrita y televisión, ofreciendo participar del estudio a mujeres que consideraran que su voz es más aguda que lo esperable. Las interesadas enviaron una muestra de audio grabada desde su teléfono que se analizó mediante el Software PRAAT(Boersma, 2001). Se seleccionaron en el grupo casos (voz hiperaguda) las voces cuya F0 estuviera sobre $250 \mathrm{~Hz}$.

Los casos fueron citados para realizar una nueva grabación de voz y aplicación de la Pauta de Evaluación de la Muda Vocal (PEMUV).

En el caso de los controles, fueron ubicados entre trabajadoras y estudiantes de la universidad donde se realizó la investigación.

Todas las mujeres del grupo caso y control fueron evaluadas por un médico otorrinolaringólogo mediante nasofibroscopía (videonasofibroscopio marca Olympus modelo ENF-VH); se excluyeron del estudio a las mujeres que no presentasen cuerdas vocales normales y a aquellas que mencionaran durante la entrevista o la anamnesis haber presentado a lo largo de su vida algún trastorno endocrino o algún trastorno de la voz de tipo orgánico o funcional. Además, se excluyeron los sujetos con entrenamiento de voz cantada o partícipes de coros. Por último, se excluyeron los pacientes que presentaran algún cuadro respiratorio agudo o crónico.

Grabación: Se grabaron los siguientes estímulos: 1) 3 vocales sostenidas /a/ para luego extraer promedio, 2) lectura meses del año 3) lenguaje espontáneo correspondiente a la respuesta a la orden: describa todo lo que hizo ayer. Esta grabación duró 1 minuto.

El micrófono utilizado fue de tipo condensador, modelo Samson C01U con una interfaz PreSonus, modelo Audio Box USB96. El micrófono se ubicó a $20 \mathrm{~cm}$ de la boca del sujeto. Además, se utilizó un atril para sostener y mantener firme el micrófono y evitar ruido asociado a movimiento o manipulación. La posición de los sujetos fue de pie durante toda la grabación de la muestra. Las muestras se grabaron en formato WAV, con una velocidad de muestreo de $44 \mathrm{KHz}$ y con 16 bits/muestra de cuantificación.

Luego de efectuadas las grabaciones se procedió a realizar el análisis muestral con el programa PRAAT versión 5.2.01. 


\section{Medición de variables}

\section{Variables objetivas cuantitativas}

Se analizó la frecuencia fundamental promedio de la vocal sostenida /a/, lectura meses del año y lenguaje espontáneo. Además, con el estímulo de la vocal /a/ se analizó jitter, shimmer y harmonic noise ratio (HNR).

\section{Variables cualitativas}

Se analizaron las respuestas a las preguntas de la Pauta de Evaluación de la Muda Vocal (PEMUV) (Nercelles, 2018). Este considera aspectos como autopercepción de la voz, características de personalidad, figura paterna y rol de la familia. Su estructura presenta preguntas tipo escala de Likert, con los siguientes puntajes: nunca (0), casi nunca (1), a veces (2), casi siempre (3) y siempre (4).

Por último, se analizó cualitativamente una pregunta abierta de este cuestionario que permite valorar molestias que el sujeto presenta en relación con la voz y el trastorno vocal. Para lo anterior, se generaron códigos in vivo con todas las respuestas entregadas.

Todos los participantes tenían como lengua materna el español y firmaron consentimiento informado para participar en el estudio. Además, el proyecto fue aprobado por el Comité de Ética de Investigación de la Universidad Santo Tomás (CE UST N48/2018).

Análisis estadístico: La prueba de Levene se aplicó para establecer la igualdad de varianzas y Shapiro Wilk para la normalidad de los datos. Para las variables cuantitativas, se calcularon estadísticas descriptivas para todas las variables, incluyendo promedio y la desviación estándar. Por último, se utilizó una prueba t de muestras independientes.

Para el análisis de las variables cualitativas, se utilizó la prueba no paramétrica U de Man-Whitney. El análisis se realizó utilizando el software IBM SPSS versión 9.6.0.0.

\section{Resultados}

En primer lugar, se exponen los resultados de las variables cuantitativas. La Tabla 1 resume los valores promedios de la frecuencia fundamental para los casos y controles.

En la /a/ sostenida, en todas las emisiones los casos obtienen valores sobre los $250 \mathrm{~Hz}$. Sin embargo, los valores se hacen más agudos en la emisión de lenguaje espontáneo. Al comparar los resultados de F0 para /a/ sostenida, lenguaje automático y lenguaje espontáneo entre los casos y los controles, se obtienen diferencias significativas entre ambos grupos.

Tabla 1.Valor promedio de F0 para casos y controles en las distintas vocalizaciones

\begin{tabular}{lccc}
\hline Vocalización & Promedio F0 Caso & Promedio F0 Control & /p/ \\
\hline A sostenida & $259.33 \pm 9.92$ & $209.59 \pm 19.83$ & 0.002 \\
Lenguaje automático & $257.56 \pm 6.38$ & $205.32 \pm 13.02$ & 0.003 \\
Lenguaje espontáneo & $268.78 \pm 14.73$ & $213.29 \pm 12.93$ & 0.002 \\
\hline
\end{tabular}

F0: Frecuencia Fundamental

La Tabla 2, muestra la comparación de los promedios de los parámetros de perturbación y ruido obtenidos para casos y los controles. Al analizar los parámetros de perturbación en A prolongada, se observó que tanto casos como controles obtienen valores dentro de la norma. Se encuentran diferencias significativas en jitter y shimmer entre los dos grupos.

Tabla 2. Valor promedio de los parámetros de perturbación y ruido en casos y controles

\begin{tabular}{lccc}
\hline A sostenida & Caso & Control & $/ \mathbf{p} /$ \\
\hline Jitter & $0.51 \pm 0.30$ & $0.47 \pm 0.15$ & 0.05 \\
Shimmer & $0.51 \pm 0.30$ & $0.62 \pm 0.27$ & 0.00 \\
HNR & $20 \pm 8.29$ & $16 \pm 3.20$ & 0.11 \\
\hline
\end{tabular}

HNR: Harmonic Noise Ratio 
La Tabla 3 presenta la media, desviación estándar y valor /p/ por pregunta del PEMUV para los casos y los controles. Al comparar los valores obtenidos entre los casos y los controles, se observó que en la mayor parte de las preguntas las diferencias entre ambos grupos son significativas, con excepción de la pregunta 4 "Siento molestias al hablar" $(\mathrm{p}=0.29)$ y la pregunta 13 "Me considero una persona tímida" $(\mathrm{p}=0.61)$.

En el grupo casos, las dos preguntas con mayor puntaje fueron la número 2 "no me gusta mi voz", y la número 12 "Tienden a confundirme con alguien menor en edad". Las preguntas con menor puntaje fueron la número 14 "Siento que tengo problemas de identidad sexual" y la número 5 "Cuando hablo mucho voy perdiendo mi voz", con una suma de 13 puntos.

Para los controles, las preguntas con mayor puntaje fueron la número 1 "presento problemas en mi voz" con una suma de 16 puntos y la número 5 "Cuando hablo mucho voy perdiendo mi voz" con una suma de 11 puntos en total. Las preguntas con menor puntaje fueron la número 14 "Siento que tengo problemas de identidad sexual” y la número 9 "Me siento avergonzada cuando me pide que repita lo dicho".

Tabla 3. Valores correspondientes a la media por pregunta del PEMUV correspondiente a casos y controles

\begin{tabular}{|c|c|c|c|c|}
\hline & Preguntas & Media Control & Media Casos & $/ \mathbf{p} /$ \\
\hline 1 & Presento problemas en mi voz. & $0,67 \pm 1,02$ & $2,83 \pm 0,56$ & 0,00 \\
\hline 2 & No me gusta mi voz. & $0,25 \pm 0,66$ & $3,58 \pm 0,44$ & 0,00 \\
\hline 3 & Me esfuerzo mucho para hablar. & $0,29 \pm 0,11$ & $2,16 \pm 0,46$ & 0,00 \\
\hline 4 & Siento molestias al hablar. & $0,33 \pm 0,38$ & $2,16 \pm 0,56$ & 0,29 \\
\hline 5 & Cuando hablo mucho voy perdiendo mi voz. & $0,45 \pm 1,08$ & $1,08 \pm 0,65$ & 0,00 \\
\hline 6 & $\begin{array}{l}\text { La calidad de mi voz es impredecible, especialmente } \\
\text { cuando grito o estoy nerviosa, me sale otra voz. }\end{array}$ & $0,37 \pm 1,27$ & $3 \pm 0,57$ & 0,00 \\
\hline 7 & Mi mamá o la persona que me crió es sobreprotectora. & $0,41 \pm 1,46$ & $2,16 \pm 0,65$ & 0,00 \\
\hline 8 & $\begin{array}{l}\text { Me molestan por mi voz, mis compañeros se burlan } \\
\text { de mí. }\end{array}$ & $0,12 \pm 0,95$ & $3 \pm 0,33$ & 0,00 \\
\hline 9 & $\begin{array}{l}\text { Me siento avergonzada cuando me piden que repita } \\
\text { lo dicho. }\end{array}$ & $0,08 \pm 0,93$ & $3,16 \pm 0,28$ & 0,00 \\
\hline 10 & Uso el teléfono menos de lo que debería. & $0,16 \pm 1,82$ & $2,33 \pm 0,38$ & 0,00 \\
\hline 11 & Tiendo a evitar conversaciones debido a mi voz. & $0,12 \pm 1,46$ & $1,83 \pm 0,33$ & 0,00 \\
\hline 12 & Tienden a confundirme con alguien menor en edad. & $0,16 \pm 0,79$ & $3,33 \pm 0,38$ & 0,00 \\
\hline 13 & Me considero una persona tímida. & $0,25 \pm 0,98$ & $2,58 \pm 0,44$ & 0,61 \\
\hline 14 & Siento que tengo problemas de identidad sexual. & 0 & 0 & 0,00 \\
\hline \multicolumn{2}{|r|}{ Suma } & 3,7 & 33,2 & \\
\hline \multicolumn{2}{|c|}{ Promedio } & 0,26 & 2,37 & \\
\hline
\end{tabular}

La Tabla 4 resume el listado de códigos in vivo que se generaron al analizar la pregunta abierta del PEMUV.

Tabla 4. Lista de códigos in vivo, análisis de la pregunta abierta de la Pauta de Evaluación de la Muda Vocal (PEMUV)

\begin{tabular}{lcc}
\hline Código & Caso & Número de ocurrencia \\
\hline $\mathbf{1}$ & Familiar & 3 \\
$\mathbf{2}$ & Laboral / educacional & 9 \\
$\mathbf{3}$ & Sentimientos negativos (malestar, inseguridad, vergüenza, & 7 \\
$\mathbf{4}$ & frustración y baja autoestima) & 6 \\
\hline
\end{tabular}

A continuación, se muestran transcripciones de los códigos y la concurrencia de los mismos.

- Código 2 (Caso 3): "En la práctica profesional el jefe de la corporación me discriminó por mi voz, y me expulsó de la práctica, así que no pude realizarla".

- Código 4-2-3 (Caso 7): "Por mi voz confunden la edad que tengo, sobre todo en el trabajo, no me toman en serio. Me gustaría sentirme más segura al hablar”. 
- Código 1-2-4 (Caso 8): "Problemas en mis relaciones familiares y el trabajo, tienden a no respetarme porque hablo como niñita".

- Código 2-4 (Caso 10): "Cuando iba en el colegio no me gustaba hacer disertaciones y ahora en el trabajo evito lo más posible hablar en público y hablar por teléfono, siempre piensan que soy menor de edad".

- Código 4-3 (Caso 12): "Tengo voz de niña chica, y siempre cuando hablo me miran y soy mamá de 2 niñas, no me gustaría que les dijeran a ellas como habla su mamá. Me avergüenza".

\section{Discusión}

El objetivo de esta investigación fue establecer un perfil vocal de mujeres con voces hiperagudas y determinar las molestias psicosociales que puede desencadenar este síntoma comparándola con mujeres con voz con frecuencia fundamental normal.

En este estudio se describen los sentimientos negativos y problemas en las relaciones familiares, laborales y sociales que presentan las mujeres adultas con voz hiperaguda. Estos resultados son similares a los obtenidos en investigaciones con varones que establecen una relación entre la puberfonía con la baja autoestima, dificultades a nivel laboral y de convivencia social (Andrews \& Summers, 2002; Anelli et al., 1999; Bhattarai, Shrestha, \& Shah, 2010; Cielo, Beber, Maggi, Körbes, Oliveira, Weber, \& Tusi, 2009).

Los valores cuantitativos en la medición de las voces, demuestran que sólo se encontró alterada la frecuencia fundamental. Los parámetros de distorsión de Jitter, Shimmer y HNR se encontraron dentro de la norma. Cabe destacar que si hubo diferencias significativas entre ambos grupos. Estos resultados no coinciden con otras investigaciones en varones que refieren valores de RAP, Shimmer y ruido alterados en sujetos con puberfonía (Edwards, 2007).

Algunos autores postulan que el cambio de la voz en la mujer es mínimo, por lo que un tono de voz más agudo en la adultez puede pasar desapercibido incluso para ellas mismas. (Aponte, 2003; Brosé, 2018). En este estudio se evidencia una dificultad importante en las mujeres evaluadas, quienes refieren burlas de familiares, compañeros de estudio y trabajo, lo que desencadenó sentimientos de vergüenza y baja autoestima.

En el caso de mujeres más adultas que se encuentran insertas en el mundo laboral se identificó el sentimiento de frustración, al sentir que sus jefes o compañeros de trabajo no las respetaban. Por lo que se concluye en esta investigación que las mujeres con voces hiperagudas, sí son conscientes de que poseen una voz diferente y que esta condición provoca menoscabos en su esfera psicosocial. Según la autora Patricia Neto, ocurre algo similar en varones con voz aguda, estos sujetos tienen dificultades en sus trabajos debido a que la voz les hace proyectar una imagen frágil y sin autoridad. Además, la voz aguda se asociaría a personalidad insegura y baja autoestima, aspectos que muchas veces son prioritarios en el ámbito laboral (Neto, 1999).

Las preguntas del PEMUV con más puntaje fueron "No me gusta mi voz" y "Tienden a confundirme con alguien menor en edad". Además, en la pregunta abierta se pudieron demostrar la presencia de algunos de estos sentimientos de inseguridad y ansiedad, así como de frustración y baja autoestima (código 3), también se demostró la crisis de identidad infantil versus adulto (código 4). Lo anterior concuerda con otros estudios que refieren que el tener una voz aguda provoca sentimiento de rechazo, agresividad, ansiedad, hostilidad, crisis de identidad infantil versus adulto y dificultad para expresar estos sentimientos (Anelli et al., 1999; Marinho, 1999).

\section{Conclusiones}

La presente investigación permitió establecer un perfil acústico y psicosocial de mujeres con voces hiperagudas. Los instrumentos utilizados resultaron sensibles para valorar los cambios acústicos y las molestias psicosociales que pueden desencadenar esta alteración en los sujetos que la padecen. De acuerdo con los resultados obtenidos, las mujeres con voz hiperaguda, presentan valoración negativa de la voz, molestias a nivel emocional y social.

\section{Bibliografía}

Andrews, M. L., \& Summers, A. C. (2002). Voice treatment for children and adolescents: United Nations Publications.

Anelli, W., Costa, H., Duprat, A., \& Eckley, C. (1999). Entendendo a muda vocal. Laringologia pediátrica. Sao Paulo: Roca.

Aponte, C. (2003). Evolución de la voz desde el nacimiento hasta la senectud. Acta otorrinolaringol. cir. cabeza cuello, 31(2, supl), 44-48.

Banerjee, A., Eajlen, D., Meohurst, R., \& Murty, G. (1995). Puberphonia-A Treatable Entity. Paper presented at the 1 World Voice Congress Oporto, Portugal.

Behlau, M. (1995). Avaliação e tratamento das disfonias. Sao Paulo: Editora Lovise.

Bhattarai, B. B., Shrestha, A., \& Shah, S. K. (2010). Psychosocial impact on puberphonic and effectiveness of voice therapy: a case report. Journal of College of Medical sciences, 6(1), 57-62.

Boersma, P. W., \& David. (2001). Praat: doing phonetics by computer. Glot International, 5(9), 341-345. 
Brosé, D. (2018). La puberfonía. Tratamiento logopédico de un caso concreto. (Logopedia), Universidad de Valladolid.

Cielo, C. A., Beber, B. C., Maggi, C. R., Körbes, D., Oliveira, C. F., Weber, D. E., \& Tusi, A. R. (2009). Disfonia funcional psicogênica por puberfonia do tipo muda vocal incompleta: aspectos fisiológicos e psicológicos. Estudos de Psicologia (Campinas), 26, 227-236.

Colton, R., Casper, J., \& Leonard, R. (2011). Understaniding voice problems: A Physiological Perspective for Diagnosis and Treatment: Wolters Kluwer Health Adis.

Denizoglu, I. I., Sahin, M., Bayrak, S., \& Uygun, M. N. (2018). Efficacy of Doctorvox Voice Therapy Technique for Mutational Falsetto. Journal of Voice, 32(4). doi: https://doi.org/10.1016/j.jvoice.2018.05.012

Dinville. (1996). Los trastornos de la voz y su reeducación. Barcelona: Masson.

Edwards, D. (2007). Puberphonia. Canadian Music Educator / Musicien Educateur au Canada, 48(4), 54-54.

Fuchs, M., Fröehlich, M., Hentschel, B., Stuermer, I. W., Kruse, E., \& Knauft, D. (2007). Predicting mutational change in the speaking voice of boys. Journal of Voice, 21(2), 169-178.

Liang, F. Y., Huang, X. M., Chen, L., Huang, Y. Z., Zhang, X. Y., Su, J. H., . . . Guan, Z. (2017). Voice Therapy Effect on Mutational Falsetto Patients: A Vocal Aerodynamic Study. Journal of Voice, 31(1), 114.e111-114.e115. doi: 10.1016/j.jvoice.2016.03.017

Marinho, A. (1999). Disfonias e alterações hormonais In H.O. Costa, A.C. Duprat, C.A. Eckey. Laringologia pediátrica (pp. 2338). Sao Paulo: Roca.

Nercelles, L. (2018). Perfil vocal y psicosocial de los sujetos con puberfonía. Revista de Logopedia, Foniatría y Audiología, $38(2), 45-51$.

Nercelles, L. (2019). Avaliação e tratamento da puberfonia: revisão da literatura. Adolesc Saude, 16(1), 103-112.

Neto, P. (1999). Muda vocal refletindo sobre a imagem vocal do adolescente Rio de janeiro.

Remacle, M., Matar, N., Verduyckt, I., \& Lawson, G. (2010). Relaxation thyroplasty for mutational falsetto treatment. Annals of otology, rhinology and laryngology., 119(2), 105-109. doi: 10.1177/000348941011900207

Spiegel, J. R., Sataloff, R. T., \& Emerich, K. A. (1997). The young adult voice. Journal of Voice, 11(2), 138-143.

Wojciechowska, A., Obrebowski, A., Studzinska, K., \& Swidzinski, P. (2010). [Mutation voice disorders conditioned by psychic factors]. Otolaryngologia Polska, 64(1), 51-54. doi: 10.1016/s0030-6657(10)70036-6 